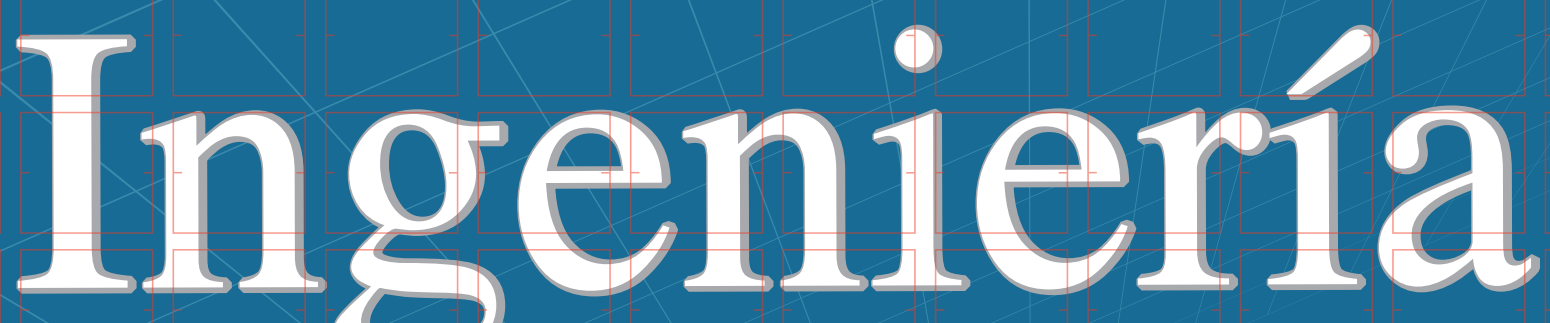

Revista de la Universidad de Costa Rica AGOSTO/DICIEMBRE 2012 - VŌLUMEN 22 Número (2)

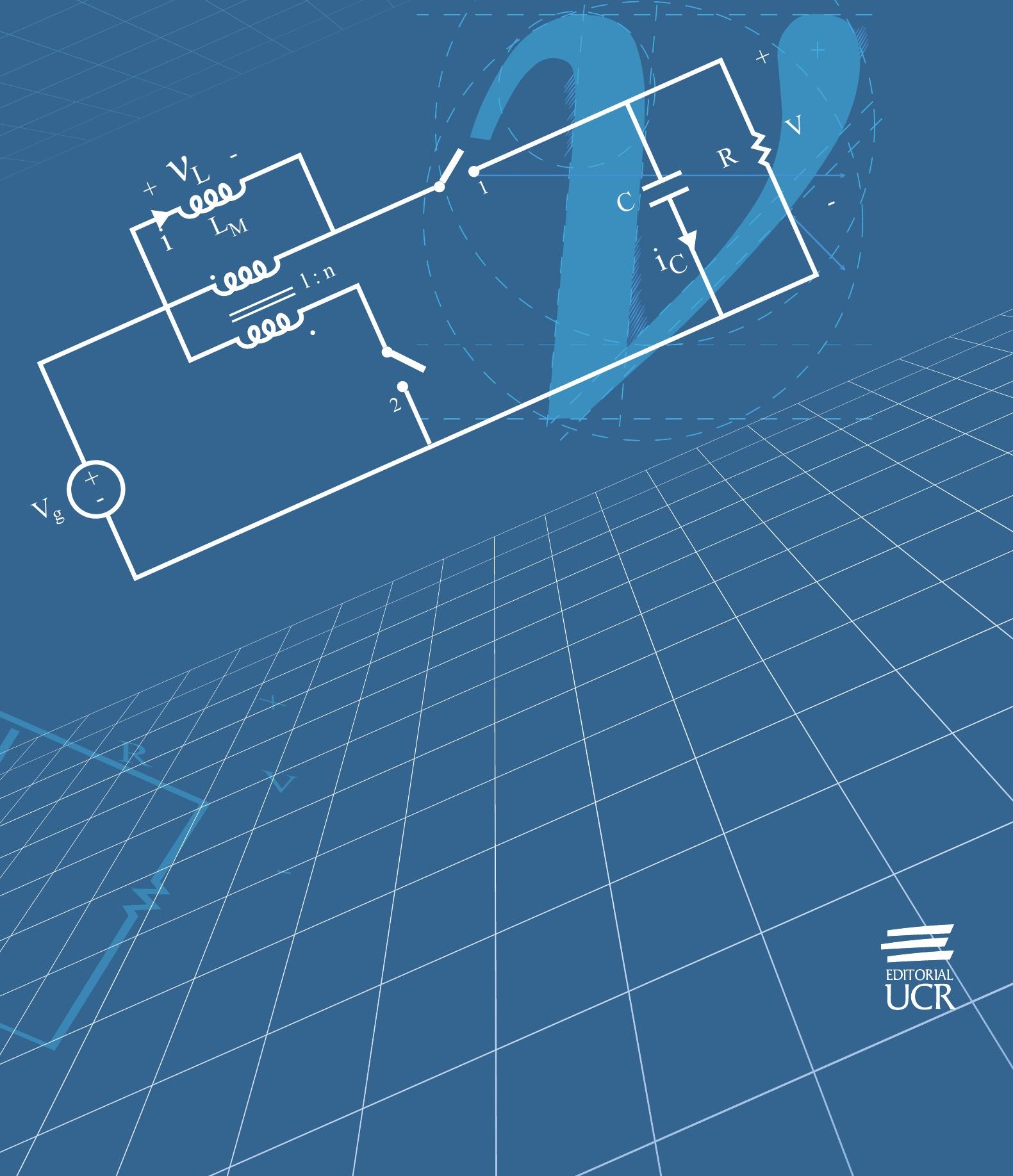




\section{Ingeniería}

Revista Semestral de la Universidad de Costa Rica Volumen 22 - Agosto/Diciembre 2012 -Número 2

\section{DIRECTOR}

Ing. Juan Carlos Chaves De Oña, Ph. D. Escuela de Ingeniería Mecánica Universidad de Costa Rica

\section{EDITOR}

Ing. Manuel Montoya Maroto. Escuela de Ingeniería Industrial Universidad de Costa Rica

\section{CONSEJO EDITORIAL}

Ing. Edwin Solórzano Campos, M. Sc. Decano de la Facultad de Ingeniería, Universidad de Costa Rica Ing. Georges Govaere Vicarioli, Ph. D. Director del Instituto de Investigaciones en Ingeniería, Universidad de Costa Rica Ing. Ronald Argüello Venegas, Ph. D. Profesor pensionado de la Universidad de Costa Rica

Ing. Juan Gabriel Monge Gapper, M. Sc., Profesor de la Escuela de Ingeniería Mecánica, Universidad de Costa Rica Ing. Guillermo Santana Barboza, Ph. D. Profesor de la Escuela de Ingeniería Civil, Universidad de Costa Rica Ing. Juan Araque Skinner, M.Sc. Director de la Escuela de Ingeniería Topográfica, Universidad de Costa Rica

Ing. Manuel Molina Córdoba. Profesor de la Escuela de Ingeniería Química, Universidad de Costa Rica Ing. José Miguel Mena Marín, MBA, GIP LTDA

Ing. Luis Diego Marín Naranjo, M. Sc., Profesor de la Escuela de Ingeniería Eléctrica, Universidad de Costa Rica Ing. Rodolfo Herrera Jiménez, Ph. D. Profesor emérito de la Escuela de Ingeniería Civil, Universidad de Costa Rica

\section{CORRESPONDENCIA Y SUSCRIPCIONES}

Editorial de la Universidad de Costa Rica Apartado Postal 11501-2060

Revista Semestral de la Universidad de Costa Rica

\section{CANJES}

Universidad de Costa Rica

Sistema de Bibliotecas, Documentación e Información

Unidad de Selección y Adquisiciones-CANJE

Ciudad Universitaria Rodrigo Facio Brenes

Costa Rica

\section{SUSCRIPCIÓN ANUAL:}

Costa Rica: $₫ 4000,00$

Otros países: US\$20,00

\section{NÚMERO SUELTO:}

Costa Rica: $₫ 2000,00$

Otros países: $\$ 20,00$

Esta revista se encuentra indizada en Latindex,

Engineering Index y Compendex 


\section{Ingeniería}

Revista Semestral de la Universidad de Costa Rica

Volumen 22 - Agosto/Diciembre 2012 -Número 2

Edición aprobada por la comisión de la Universidad de Costa Rica

(C) 2010 EDITORIAL UNIVERSIDAD DE COSTA RICA

Todos los derechos reservados conforme a la ley

Ciudad Universitaria Rodrigo Facio Brenes

San José, Costa Rica

\section{Supervisión:}

Ing. Juan Carlos Chaves De Oña, Ph.D.

Ing. Manuel Montoya Maroto, E.I.

D.I. Marcela Quirós Garita, M.Sc.

\section{Colaboración:}

Srta. Ingrid Vargas Romero, asistente de filología, estudiante de la Escuela de Filología de la Universidad de Costa Rica.

Srta. Ana Elizabeth González López, estudiante de la Escuela de Arquitectura de la Universidad de Costa Rica.

\section{Diagramación:}

Luis Arias Mesén.

Katherine Canales Montoya, estudiante de Artes Plásticas con énfasis en Diseño Gráfico de la Univesidad de Costa Rica.

\section{Diseño de portada:}

Luis Arias Mesén. 


\section{Proyectos de Graduación}




\title{
LISTA DE PROYECTOS DE GRADUACIÓN DE GRADO Y POSGRADO DEL AÑO 2011 DE LA FACULTAD DE INGENIERÍA DE LA UNIVERSIDAD DE COSTA RICA.
}

\author{
ESCUELA DE INGENIERÍA CIVIL
}

\section{GRADO DE LICENCIATURA}

Agüero Maroto, Brandon. Secuencia de falla a flexión y a cortante en muros de mampostería sometidos a fuerza lateral

Aguilar Oreamuno, Manrique. Análisis constructivo de nueva técnica para colocación de sisas verticales en paños de mampostería hueca.

Alfaro Gómez, William. Aplicación de conceptos de administración profesional de proyectos en la construcción de pisos planos y superplanos en Costa Rica.

Alfaro Solís, Felipe. Control de costos de obra con la aplicación de la guía del PM$\mathrm{BOK}{ }^{\circledR}$ para la gestión de los costos del proyecto en las obras de contrapresa, en el Proyecto Hidroeléctrico Pirris.

Araya Mendoza, Gabriela. Diagnóstico de la planta potabilizadora de Las Juntas de Abangares.

Arroyo Castro, Houseman Miguel. Análisis de estabilidad de taludes en suelos cohesivos considerando los desplazamientos.

Bermúdez Mora, Silvia. Análisis y propuesta para el mejoramiento del sistema de alcantarillado sanitario del distrito de San Rafael de Oreamuno.

Brenes Rodríguez, Erick. Diagnóstico y recomendaciones para el manejo de residuos sólidos en el cantón de Palmares.

Brizuela Elizondo, José Pablo. Metodología para el diseño de dispositivos disipadores de energía en sistemas pluviales de carreteras.

Calcáneo Maduro, Felipe. Homologación del cálculo de la demanda sísmica para Costa Rica con el manual de cargas mínimas de diseño área edificios y otras estructuras de la Sociedad Americana de Ingenieros Civiles 2010 (ASCE-07-2010) 
Calderón Moya, Marvin; Arias Carballo, Priscilla. Diseño de una planta de tratamiento de aguas para un centro hospitalario.

Centeno Mora, Erick. Análisis comparativo de distintas tecnologías de tratamiento de aguas para municipalidades de Costa Rica.

Chanto Ballestero, Gabriela. Análisis comparativo de distintas tecnologías de tratamiento de aguas para municipalidades de Costa Rica.

Corrales Chacón, Emilio. Dimensionamiento preliminar de la planta de tratamiento de aguas residuales para la Urbanización Jorge de Bravo y sus alrededores, en el distrito de Turrialba.

Corrales Martínez, Juan Carlos. Listas de verificación y guía para la obtención de permisos de construcción y operación de proyectos de tipo industria.

Elgarrista Rodríguez, Laura Alejandra. Análisis de factibilidad de sistemas con las cosas postensadas de entrepiso como parte de sistemas sismo resistentes.

Esteban Zander, David. Análisis de la aplicación de construcción flexible a las diferentes fases del proyecto.

Fallas Barrantes, Fernando. Aplicación del método de reflectometría en el dominio de tiempo para el análisis y monitoreo de la estabilidad de taludes.

Fernández Segura, Denia. Guía práctica para control de erosión superficial de taludes usando vegetación nativa de Costa Rica.

González Amón, Luis Esteban. Lista de verificación y guía técnica para permisos de construcción y operación de proyectos de carácter turístico en Costa Rica.

Grant Alpízar, Karla Jimena. Determinación de la respuesta sísmica de puentes sesgados de dos claros con libertad de movimiento en los bastiones.

Guerrero Vargas, Christian. Determinación de ecuaciones para modulo de elasticidad en concreto elaborados en planta con resistencias mayores a $50 \mathrm{MPa}$, en Costa Rica.

Hidalgo Miranda, Deilyn. Listas de verificación y diagnostico de tramitología aplicable a permisos de construcción para plantas de tratamiento de aguas residuales

Huguenin Amador, Caroline Noor. Análisis de estabilidad de los terraceos como solución para los deslizamientos en la Ruta Nacional 126, sector Vara Blanca.

Jiménez Arias, Reiner Antonio. Propuesta de mejoras ante riesgo por incendio y aplicación de la ley 7600 en las instalaciones del Liceo de Poás, Alajuela. 
Jiménez Molina, Daniel. Análisis del efecto de cargas axiales sobre el comportamiento de paredes de mampostería confinada en escala reducida.

Leitón Montero, Juan. Cambio en el patrón de la escorrentía superficial de la cuenca alta del rio Tempisque ante diferentes escenarios de cambio climático.

Leiva Cordero, Ana Lorena. Estudios de las causas de la generación de desechos (concreto, madera y acero) en la construcción de vivienda tradicional.

Molina Quesada, Grettel. Vulnerabilidad sísmica funcional de la red vial del Área Metropolitana de San José.

Molina Zamora, Doris. Evaluación de la cobertura y calidad de la desinfección en los acueductos comunales de la provincia de Cartago.

Mora Castillo, Carlos Andrés. Análisis de la capacidad al cortante en vigas con secciones rectangulares compuestas de concretos de diferente resistencia.

Mora Castro, Susan. Evaluación de las principales tecnologías utilizadas en Costa Rica para la desinfección del agua.

Murillo Carranza, Luis Ricardo. Listas de verificación y guía técnica para la obtención de permisos de construcción en condominios de lotes.

Oconitrillo Fuentes, Ana Victoria. Análisis de soporte utilizado para el túnel de conducción del PH Paso Ancho, Volcán Panamá.

Quesada Sandí, Allan Humberto. Determinación de una herramienta numérica para el análisis de consolidación primaria en suelos finos saturados.

Rodríguez Jiménez, Marco Augusto. Propuesta de normativa para el diseño hidráulico de obras de protección de cauces.

Sancho Arias, José Daniel. Modulación de una vivienda de interés social para diferentes sistemas constructivos.

Solano Arguello, Luis Martin. Validación de características dinámicas de un edificio tipo dual, en acero con programas de cómputo.

Solano Ramírez, Rally María. Estrategias de climatización pasiva aplicadas al Hospital Dr. Max Terán Valls, ubicado en Quepos de Aguirre en la zona de vida bosque húmedo tropical

Sotela Sobrado, Cristian. Zonificación de sitios para valoración de la aceleración pico efectiva según criterios del Código Sísmico de Costa Rica. 
Suárez Barboza, Lourdes. Evaluación del uso de la incineración como solución para el tratamiento de los desechos sólidos en Costa Rica.

Vargas Chacón, Sergio. Plan de proyecto para la construcción de centros educativos, según estándares del PMI.

Vega Díaz, Eduardo. Evaluación de la capacidad de explotación del sistema de conducción y almacenamiento y de la calidad de agua suministrada del Acueducto de la Isla Chira para tres escenarios de crecimiento.

Venegas Sáenz, Diana. Caracterización de la deformabilidad y resistencia de suelos lateríticos en tres puntos de la nueva carretera San Ramón -San Carlos.

Villalobos Cardozo, Uver Darío. Comportamiento mecánico de unión universal en un sistema constructivo de bambú Gadua.

Zamora Montoya, Javier. Diseño hidráulico de concretos permeables

\section{ESCUELA DE INGENIERÍA ELÉCTRICA}

\section{GRADO DE LICENCIATURA}

Araya Harley, Ismael. Diseño de un controlador gráfico en LabVIEW ${ }^{\mathrm{TM}}$ para el Staubli.90®.

Arguello Leandro, José Pablo. Diseño e implementación de un sistema de comunicación mediante el parpadeo voluntario del usuario.

Arias Espinoza, José Gabriel. Estudio de las cargas eléctricas y levantamiento de planos del departamento de metrología física del Lacomet.

Barrantes Chaves, Emmanuel. UCRASM entorno de diseño de programación para ensamblador NASM con el set de instrucciones IA32.

Barrantes Chaves, Mariana. Estimación y análisis de la cargabilidad de transformadores de distribución residenciales mediante la utilización de ArcGIS ${ }^{\circledR}$ y ArcFM ${ }^{\circledR}$.

Bustos Torres, Hernan. Arbitraje en el bus del sistema multiprocesador GPU theia

Castro Chan, Ricardo. Validación del software de simulación "OpenDSS" para su aplicación en sistemas de distribución eléctrica del Instituto Costarricense de Electricidad (ICE). 
Campos Pérez, Pedro. Validación método macroeconómico basado en la disposición a para por el cliente, para el cálculo del costo de energía no servida residencial (CENSR), en la zona servida por la CNFL.

Castro Chan, Ricardo. Validación del software de simulación "OpenDSS" para su aplicación en sistemas de distribución eléctrica del Instituto Costarricense de Electricidad (ICE).

Chinchilla Vargas, Erick. Diseño de un sistema de control de dosificación de cloro en el acueducto rural de San Gabriel de Aserrí.

Espinoza Rivero, Javier Francisco. Modernización de los sistemas de control de la unidad \# 1 del Centro de Producción Toro II.

Fernández Otárola, Marcos Esteban. Implementación de un sistema de adquisición y análisis para el laboratorio de maquinas y eléctricas I mediante el software LabVIEW.

Fernández Rodríguez, Berny. Obtención del costo de energía no servida comercial par la CNFL utilizando métodos macroeconómicos.

Garita Rodríguez, Esteban José. Implementación de un interfaz cerebro computador utilizando hardware y software libres.

Marín Morales, Mario. Implementación del programa de simulación optiwave para el diseño de redes de comunicación ópticas en LAFTLA.

Pérez Arroyo, David José. Estudio e implementación de amplificador de audio de tipo AB.

Pérez Sánchez, Daniel Antonio. Factibilidad para implementar un laboratorio de energía eólica en la Escuela de Ingeniería Eléctrica(2010).

Rodríguez Brenes, Daniel. Análisis de robustez para un sistema de control difuso aplicado a sistemas de primer y segundo orden.

Rodríguez Castillo, Francisco. Calidad de servicio en la interfaz de radio en redes UMTS.

Rodríguez Ordoñez, Jimmy. Desempeño de la técnica OFDM sobre canales inalámbricos.

Sánchez Vargas, Jesús Leonel. Descripción del funcionamiento de un módulo clasificador de ondas electroencefalografías utilizando la plataforma Open Vibe.

Soto Leandro, Felipe; Vásquez Castillo, Alex. Comparación técnica y en financiera entre el desarrollo de redes de distribución eléctrica subterráneas y aéreas de tipo residencial.

Torres Romero, Erick. Generación distribuida: estudio de requerimientos para la interconexión a la red de distribución. 
Valverde Zúñiga, Gabriel. Diseño de una aplicación para el iphone ${ }^{\circledR}$ para el control del Staubli ${ }^{\circledR}$ RX90 L.

Valverde Monge, Mauricio. Desarrollo de aplicaciones en Google Android. Desarrollo de la bolsa de empleo para la Escuela de Ingeniería Eléctrica con Ruby on Rails.

Vargas Piedra, Francisco Andres. Análisis y comparación de métodos de medición del desempeño de redes de computadores ethernet y metro Ethernet.

Víctor Soto, Luis Alberto. Tecnología de voz sobre LTE, un paso hacia el futuro telefónico.

Vieto, Ignacio. Simulación del desempeño de un motor de inducción trifásico operado por una fuente monofásica y compensada por un convertidor de fase estático.

Villalobos Fernández, Marcelo; Magaña Godoy, Saúl; Solís Carballo, Emmanuel. Uso de energías renovables no contaminantes para alimentación de estaciones a base de sistemas de telecomunicaciones móviles en Costa Rica.

Díaz Bolaños, Daniel Antonio. Extensión de blender para la generación de archivos de entrada de simulación de escenas tridimensionales del GPU Theia.

Dumani Jarquín, Diego Sayid. Desarrollo de procedimientos de adquisición y procesamiento de señales electroencefalográficas utilizando el hardware libre Open EEG y el software libre Open Vibe.

Elizondo Segura, Marcia Pamela. Estudio de la interconexión de la primera ampliación de la central generadora las Pailas al Sistema Eléctrico Nacional.

Estrada Garzona, Ercilia. Interfaz de diseño para cargas residenciales utilizando Guía de Matlab.

Fernández Mora, Luis Carlos. Tecnologías de banda ancha para la convergencia de aplicaciones: FTTx y LTE.

Gamboa Alvarado, José Leandro. Desarrollo de programas de comunicaciones para un sistema inteligente de control de iluminación.

Gatjens Villalobos, Pablo Antonio. Procedimientos para la sintonización de los dispositivos de ajuste de los dispositivos de ajuste de los reguladores automáticos de tensión de los sistemas de excitación; IEEET1, ESAC8B y ESST4B.

González Alfaro, Jeffrie Alexander. Sistemas de excitación en unidades de generación.

Guillén Hernández, Sergio Jesús. Desarrollo y validación de una herramienta para la medición del balance postural estático en personas adultas mayores utilizando la 
plataforma de balance del Nintendo Wii.

Gutiérrez López, Julio. Implementación de módulos de modulación en fase y frecuencia para la transmisión de datos.

Gutiérrez Monge Julían. Diseño e implementación de un sistema de control remoto con sensores de movimiento y rotación utilizando el protocolo de comunicación inalámbrica ZigBee.

López Leiva, Laura. Análisis de la interconexión de proyectos eólicos a redes de distribución.

Méndez Murillo, Andrés Esteban. Diseño eléctrico industrial observatorio sismológico y vulcanológico Arenal y Miravalles.

Montero Ramírez, Gustavo. Implementación de un ensayo de eficiencia para motores eléctricos de inducción en el laboratorario de eficiencia energética del ICE.

Quesada Carranza, René. Cifrado de datos mediante el uso de hardware.

Retana Barrantes, Silvia. Diseño de algoritmos de control para simuladores de procesos de eventos discretos.

Robelo Mojica, Nelson. Estudio de campos magnéticos y eléctricos en las líneas de trasmisión mediante un programa de simulación por computadora. FORMATO ELECTRONICO. DISPONIBLE EN EL RIINI

Rodríguez Arguedas, Eduardo; Bustillo Piedra, Marvin; Heilbron Carazo, Oscar Martín. Guía telefónica de mantenimiento predictivo en motores trifásicos de corriente alterna y transformadores de distribución para uso industrial.

Rodríguez Paniagua, Gerson Antonio. Diseño de circuito transformador de pulso cuadrado usando bloques de filtros de capacitor conmutado y filtros analógicos integrados.

Rojas Campos, Daniel. Implementación de un sistema de adquisición y análisis para los laboratorios de máquinas eléctricas II mediante el software LabVIEW.

Rojas Chaves, José Martín. Desarrollo de un visualizador de ondas digitales como una aplicación web.

Salvatierra Picado, Antonio Jesús. Implementación y análisis de un codificador de voz humana utilizando el algoritmo de predicción lineal.

Solano Sánchez, Kenneth. Estudio y análisis de alto consumo de energía eléctrica en residencias la zona de Juan Viñas y Turrialba. 
Solano Rivera, Juan Diego. Estudio y documentación complementaria de la condición del suministro de potencia eléctrico del manejador de lámparas FEL del LAFTLA (Laboratorio de Fotónica y Tecnología Láser).

Solera Saborío, Eugenia. Análisis y solución al impacto de las transferencias regionales en la capacidad de trasmisión del anillo norte para el horizonte 2016-2030.

Solís Salazar, Kevin. Nuevo algoritmo de estimación del umbral local para la segmentación de aglomeraciones celulares para microscopía IN-SITU.

Suárez Hernández, Eduardo Alberto. Análisis del impacto de las componentes armónicas por el uso de bombillos fluorescentes compactos en el sector residencial.

Víquez Bolaños, Nelson; Cubero Alpízar, Carlos; Murillo González, Randall. Elaboración de la base de conocimientos actualizados para el desarrollo de proyectos de generación por energías limpias aislados o conectados a red nacional de distribución de energía eléctrica.

Villalta Romero María del Milagro. Demostraciones de electromagnetismo.

Ulate Alfaro, Jorge Arturo. Protocolo para la atención de solicitudes de estudio por alto consumo.

\section{ESCUELA DE INGENIERÍA INDUSTRIAL}

\section{GRADO DE LICENCIATURA}

Aguilar Araya, Alonso; Mora Jiménez, Maureen; Mora Porras, Anthony. Propuesta para la disminución de las fuentes de desperdicio en la planta de tallarines de la empresa Fideos precocidos de Costa Rica a partir de un enfoque de manufactura ajustada.

Aguilar Castro, Marco; Castillo Castillo, Susana; Rojas Downing, Ariana. Manejo integral de residuos sólidos en la empresa Corporación de Compañías Agroindustriales, CCA, SA División Frutas, Granos y Vegetales.

Álvarez Calvo, Rolando; Ávila Arias, Luis; Villalobos Montoya, Giselle. Gestión de entregas perfectas para la satisfacción del cliente en Coca Cola FEMSA.

Araya Rojas, Rodolfo; Solano Azofeifa, Oscar Rodolfo; Vargas Rodríguez, Luis Felipe. Modelo integral de procesos institucionales entre el Hospital Nacional de Niños y el Sistema de Atención Médico para Adultos de la CCSS para la transición niñoadolescente-adulto de las personas adolescentes con enfermedades crónicas. T.I-T.II 
Araya Sequeira, Israel; Zamora Solano, Carmen. Diseño de un sistema de gestión para la administración de bienes materiales en el Programa de Atención Integral de Salud (PAIS).

Arias Rodríguez, David; Matarrita Sequeira, Diana. Aumento de la productividad en la prestación del servicio en el taller Super Servicio la Sabana.

Bogantes Aguero, Fiorella; Flores Golfin,Diego Alberto; Quesada Webb, Ana Cristina. Diseño de un modelo de gestión del control interno para el Hospital San Vicente de Paul.

Boettger Alvarez, Carlos; Salazar Ramos, Marianela. Modelo para atacar las no capacidades en máquinas de ensamble en Samtec Costa Rica.

Briceño Cordero, Luis Diego; Elizondo Wallace. Carlos Andrés; Midence Cerdas, Rebeca. Modelo sistémico para el seguimiento de la satisfacción del cliente y la medición del desempeño organizacional en la prestación de servicios de salud del Programa de Atención Integral de Salud (PAIS).

Castro Vásquez, Johan; Santamaría García, Jefrey. Desarrollo de modelo de gestión de conocimiento y rediseño de procesos del departamento de distribución local de DHL. 2 Tomos.

Cavallini Araya, Felipe; Sánchez Mora, Ricardo. Diseño del modelo de gestión de la cadena de suministros en el Departamento de Puertas y Ventanas en Durman.

Chavarría Ulate, María José; Cordero Blanco, Melania; Ramírez Céspedes, José Pablo. Modelo de gestión de operaciones de las Estaciones de Servicio del Grupo Caribeño.

Cordero Alfaro, Carolina; Jiménez Quesada Silvia Eugenia. Modelo de dotación de recursos al Servicio de Cirugía de la CCSS.

Cortés Sandí, Natalia; Méndez Morales, Daniel. Modelo para potenciar la capacidad resolutiva en los servicios de radio diagnóstico por imégenes en la subred Hospital México -Hospital San Rafael de Alajuela -Hospital Carlos Luis Valverde Vega de San Ramón a partir de los recursos existentes.

Conejo Gutiérrez, Guillermo; Jiménez Arguedas, Hernán; Zamora Guevara, Néstor. Sistema de gestión integral para el desarrollo de proyectos en Cibertec Int.

Coto Rodríguez, José Ignacio; Garro Montero, Valeria; Hernández Fallas, Juan Miguel. Diseño de un sistema de control de la producción en la Sección de Mantenimiento Mecánico de la Compañía Nacional de Fuerza y Luz "Taller Anonos".

Dejuk Protti, Geogina; Quesada Pacheco, Jorge A; Urguellés Ulloa, Alvaro J. Diseño de un sistema de valoración del riesgo en SETENA. 
Fallas Rojas, Yarelis; Jiménez Monge, Karolina. Propuesta de un modelo conceptual y estratégico para la planificación académica institucional de la Universidad de Costa Rica para posicionar la salud como producto social.

García Zúñiga, Andrés Orlando; Salazar Obando, José Alfredo. Rediseño del servicio exprés de la cadena de farmacias del Hospital Clínica Bíblica.

Gómez Moreno, Ernesto; Quirós Rojas, Aura. Diseño de un sistema de gestión de operaciones en la red Frigorífica. T.I-T.II

Jiménez Jiménez, Diana; Lacayo Morice, Ximena. Análisis y diseño orientado a objetos del sistema de planificación, programación y control de las operaciones en la empresa Spoon.

Madrigal Vargas, Angel; Morales Barquero, Pablo; Muñoz Zúñiga, José Enrique. Desarrollo de un modelo conceptual para la prestación de servicios de atención directa a las personas con énfasis en la garantía de acceso a los servicios de salud para el Programa de Atención Integral de Salud (PAIS) T.I -T.II

Ma Lai, Ericka; Murillo Sánchez, José Pablo. Propuesta de mejora de la eficiencia operacional en los proyectos de RM Center.

Matarrita Sequeira, Diana. Aumento de la productividad en la prestación del servicio en el taller Super Servicio la Sabana.

Medina Posada, Alejandra; Solera Jiménez, Mariana. Mecanismo estratégico de articulación y coordinación de la información para el acompañamiento de la Universidad de Costa Rica en la construcción conceptual y operativa del Plan Nacional de Salud, 2010-2021.

Morales Villegas, Mariel; Zamora Hernández, Eugenia. Diseño de las operaciones para el nuevo almacén de productos fríos y acopio de frutas y verduras en el Centro de Distribución de Auto Mercado T. I -T.II

Muñoz Vargas, Andrea; Yglesias Vargas, Juan Carlos. Diseño de un modelo de procesamiento de biomasa como fuente de energía alternativa en el proceso productivo de TICOFRUT S.A. incorporando elementos de gestión ambiental.

Muñoz Zúñiga, José Enrique. Desarrollo de un modelo conceptual para la prestación de servicios de atención directa a las personas con énfasis en la garantía de acceso a los servicios de salud para el Programa de Atención Integral de Salud (PAIS). T.I-T.II

Pabón Páramo, Alejandra; Saborío Solano, Sofía. Diseño de un modelo de modelo de gestión para el Departamento de Servicio al Cliente en Correos de Costa Rica S.A. 2 Tomos

Ramírez Alfaro, Paula María. Desarrollo de un modelo de vinculación externa del área de Acción Social para la Escuela de Ingeniería Industrial. 
Seco Badilla, José Daniel. Rediseño de las operaciones de una empresa productora de helados. T.I -T.II

Sequeira Chacón Ibar; Solís Cedeño, Max. Diseño de un sistema para la gestión de la información y comunicación de los proyectos realizados por Automatización Avanzada S.A.

\section{ESCUELA DE INGENIERÍA MECANICA}

\section{GRADO DE LICENCIATURA}

Balladares Phillips, Roberto; Coto Jiménez, Jorge. Diseño de un sistema de supresión de incendios para la planta de HI-C en Coronado.

Bedoya Serrano, Marco V; Torres Contreras, Moises D; Gairud Chacon, Ricardo. Diseño de maquina cortadora de tallos de palmito para la empresa Corazón Tierno S.A.

Bernini Segura, Jorge; Ortiz Cordero, Edgar; Ríos Quesada, Ignacio. Diseño de un banco de prueba de fugas utilizando técnicas de vacío y rastreo de gases residuales.

Jiménez Alvarado, Alonso; Longhi Gutiérrez, José Alberto; Solano Aguilar, Humberto. Diseño de un sistema para almacenar, calentar y trasegar bunker en la planta de cemento de Holcim en Cartago.

Montes de Oca Miranda, Bernardo; Salazar Monge, Andy. Diseño de sistema de bobinas enfriadas para un gasificador de residuos con gas ionizado.

Perez Rodriguez, Maria de los Angeles; Gomez Arango, Pablo. Diseño de talleres de mantenimiento para el centro de para el centro de producción Cachi del Instituto Costarricense de Electricidad.

\section{ESCUELA DE INGENIERÍA QUÍMICA}

\section{GRADO DE LICENCIATURA}

Acosta Solís, Olga Lorena. Dimensionamiento de un sistema de tratamiento para el manejo y la disposición de lodos generados del tratamiento físico-químico de aguas residuales de un relleno sanitario. 
Agüero Herrera, Pamela. Evaluación de dos métodos de pretramiento y de dos tipos de celulasa en la hidrólisis enzimática del rastrojo de la piña para la producción de etanol.

Aguilar Palma, Roy. Evaluación de impacto ambiental por contaminación atmosférica para la ampliación y modernización de refinadora de petróleo.

Alfaro Pérez, Johnny Daniel. Determinación del coeficiente de película de fluidos pseudoplásticos en tanque agitados calentados con sepertín.

Alizaga Rodríguez, Marcela. Comparación de la capacidad detergente del biodiesel respecto a tres aditivos comerciales para diesel.

Arias Alfaro, Sebastián. Caracterización del comportamiento cinético de la descomposición de dos tipos de surfactantes aniónicos por ozonólisis acuosa.

Arias Carballo, Priscilla; Calderón Moya, Marvin. Diseño de una planta de tratamiento de aguas para un centro hospitalario.

Arias Chaves, Adriana Vanessa. Análisis del funcionamiento de una planta de tratamiento de aguas de proceso en un beneficio de café.

Armas González, Irene Noriko. Análisis del proceso de decoloración de bases parafínicas aceitosas regeneradas a partir de aceites usados mediante la comparación entre tres adsorbentes y la fijación de la proporción.

Balladares Phillips, Beatriz. Elaboración del manual del sistema de gestión preventiva de riesgos laborales para una empresa procesadora de nueces.

Cartín Caballero, Carlos Manuel. Evaluación del efecto de la temperatura, la concentración y el tipo de celulasas en la hidrólisis enzimática del bagazo de la caña.

Cavallini Durán, Michael. Dimensionamiento de un sistema de tratamiento de lodos en una empresa de productos alimenticios. 2010

Cisneros Vargas, Denise. Diseño de un sistema activo contra incendios para una planta de almacenamiento de GLP basado en las Normas NFPA.

Contreras Leiva, Felipe de Jesús. Prefactibilidad económica para el aprovechamiento del biogás como fuerza motriz, para calefacción, utilizando como sustrato las aguas residuales de una granja porcina.

Córdoba Pérez, Maureen Daniela. Determinación del efecto de la concentración de la base $\mathrm{NaOH}$ de la celulasa y celobiasa en la hidrólisis para la producción de etanol a partir del rastrojo de piña. 
Cordero Muñoz, Ana Marcela. Prefactibilidad técnica y económica para una planta productora de anhídrido acético en Costa Rica. 2010.

Coto Rojas, Ismael Antonio. Dimensionamiento y costeo de un equipo de separación de aceite a partir de harina de pescado.

Coyado Corrales, Olger Mauricio. Dimensionamiento construcción y puesta en marcha de una columna empacada para extracción líquido -líquido.

Cubillo Carranza, Laura. Determinación de la curva agotamiento para un conjunto de resinas de intercambio iónico para la producción de agua desionizada y reconstrucción del equipo de desionizacion de agua para el laboratorio de ingeniería química de la Universidad de Costa Rica.

Ching Vindas, Diego. Diagnóstico del plantel de RECOPE en Moín para el almacenamiento de gas natural licuado.

Flores Pujol, Alberto. Efecto de degradación mecánica y térmica del poliestireno, cuando es procesado, sobre la calidad del producto final.

García Padilla, Natalia. Prefactibilidad técnica y económica de una planta para la producción de aceites base para la fabricación de aceites lubricantes a partir de la fracción de gasóleos pesados de vacío, en el plantel de Moín de la Refinería Costarricense de Petróleo.

Hernández, Márquez, Jhina Maritza. Evaluación energética en el sistema de almacenamiento y distribución de asfalto y búnker C en el plantel del Alto de Ochomogo.

Leiva Saborío, Andrea. Obtención de pulpa a partir de raquis de palma africana por el método químico kraft .

López Rodríguez, Mariana. Efecto de la cantidad de sustrato, concentración de dióxido de carbono y ciclo de trabajo en la cinética de crecimiento de un cultivo mixto de microalgas.

Marín Guzmán, Noelia. Elaboración de un plan de manejo de desechos para una planta distribuidora de gas. 2010.

Monge Carvajal, José Manuel. Diseño de un plan de implementación de una interfaz de computadora para la unidad de destilación en el Laboratorio de Ingeniería Química.

Obando Godínez, Priscilla. Dimensionamiento de una planta de tratamiento de aguas residuales residenciales en la urbanización la Trinidad de Alajuela.

Orozco Madrigal, David Roberto. Dimensionamiento y costeo de un digestor anaerobio para el tratamiento de aguas residuales para una finca porcina. 
Oviedo Valverde, Manuel. Prefactibilidad técnica y económica para una empresa fabricante de ceras utilizadas en las tablas de surf. $<2010$.

Pérez Vargas, Silvia María. Evaluación del efecto de la concentración de sacarosa, concentración de vinazas y diferencias entre lotes de vinazas para la producción de proteína insecticida por parte de Bacilus Thuringiensis Subsp. Isaraelensis.

Sánchez Méndez, Marcela. Evaluación de la pulpa para papel obtenida a partir del raquis de palma africana (Elaeis guineensis) utilizando el método organosolvente modificado.

Sojo Allen, José Iván. Protocolo para la selección de sistemas de calentamiento de agua por efecto invernadero y por concentradores solares a escala doméstica.

Solano Castillo, Denis Alfonso. Dimensionamiento de un gasificador de lecho fluidizado para el procesamiento de biomasa de café.

Ulloa Jiménez, Silvia. Evaluación del rendimiento de la producción de biodiesel a partir del aceite de la semilla de Jatropha Curcas L.

Williams Villalta, Nathalia. Evaluación de la adición de vidrio del panel frontal de tubos de rayos catódicos en la elaboración de concreto.

Vargas Sánchez, Arnoldo. Prefactibilidad para el uso de una mezcla etanol-agua como fluido de trabajo en un ciclo termodinámico.

\section{ESCUELA DE INGENIERÍA TOPOGRÁFICA}

\section{GRADO DE LICENCIATURA}

Garbanzo León, Jaime; Vargas Vargas, Esteban. Diagnóstico sobre la afectación urbana de las nacientes del distrito San Rafael de Montes de Oca, 2011.

Hidalgo Jiménez, Rolando; Carrillo Quirós, José Manuel. Propuesta de densificación al tercer orden de la Red Geodésica Nacional en la Zona del Levantamiento Catastral de Istarú. 


\section{ESCUELA DE INGENIERÍA AGRICOLA}

\section{GRADO DE LICENCIATURA}

Guerrero Brenes, Pedro Mauricio. Análisis comparativo de la medición del contenido de humedad con los medidores "de marcas" DICKEY-John, Gehaka, Motomeo y Grainmam en frijol nacional rojo variedad Cabécar y negro variedad Guaymi

Navarro Meza,Luis. Recomendaciones para el diseño de infraestructura para plantas de acondicionamiento y empaque de productos hortifrutícolas de acuerdo a las principales normas nacionales y estadounidenses. 
